Revisión de la literatura

\title{
Capital intelectual y su relación con la innovación: una revisión de la literatura
}

\section{Intellectual capital and its relationship with the innovation: a review of the literature}

\author{
Nahuat Román, Bernardo ${ }^{1}$ y Ochoa Hernández, Magda Lizet ${ }^{2}$
}

\begin{abstract}
Resumen:
El estudio de la relación capital intelectual (CI) e innovación ha estado evolucionando de forma dinámica los últimos años, por su importancia en las organizaciones se hace necesario un análisis integral a profundidad que muestre sus tendencias, así como sus principales hallazgos. Por lo cual, este trabajo tiene como propósito abordar este vacío mediante una revisión exhaustiva de la literatura del año 2016 al año 2019. Los resultados muestran que existen países como China y España donde el estudio del fenómeno se ha focalizado y en sentido inverso, el continente africano ha permanecido prácticamente ignorado. Los estudios se han diseminado en función de las propuestas y conceptos de CI e innovación tanto clásicos como contemporáneos tomando como escenario favorito la industria manufacturera. Aunque el grueso de las evidencias muestra al CI estrechamente relacionado con la innovación no se puede ser concluyente por las discrepancias y lagunas en el conocimiento existentes.
\end{abstract}

\begin{abstract}
:
The study of the relationship between intellectual capital (IC) and innovation has been evolving dynamically in recent years, due to its importance in organizations, it is necessary to carry out an indepth comprehensive analysis that shows its trends, as well as its main findings. Therefore, the purpose of this work is to address this gap through an exhaustive review of the literature from 2016 to 2019. The results show that there are countries such as China and Spain where the study of the phenomenon has been focused and in reverse, the African continent has remained practically ignored. The studies have been disseminated based on both classic and contemporary proposals and concepts of IC and innovation, taking the manufacturing industry as a favorite scenario. Although the bulk of the evidence shows intellectual capital closely related to innovation, it cannot be conclusive due to the existing discrepancies and gaps in knowledge.
\end{abstract}

Keywords: Intellectual capital, innovation, competitive advantage, organizations, trends. ción, ventaja competitiva, organizaciones, tendencias.

\footnotetext{
${ }^{1}$ Estudiante de Doctorado en Gestión Estratégica de Negocios. Reconocido en el Programa Nacional de Posgrados de Calidad (PNPC) CONACYT. Universidad Autónoma de Tamaulipas. Facultad de Comercio y Administración de Tampico.

${ }^{2}$ Profesor investigador de tiempo completo. Universidad Autónoma de Tamaulipas. Facultad de comercio y administración de Tampico
}

*Correspondencia: bernienahuat@gmail.com 
Factores de cambio en los mercados como la globalización, la competencia intensa, los avances tecnológicos, entre otros han generado un entorno incierto para las organizaciones (Buenechea-Elberdin, Sáenz y Kianto, 2017) obligándolas a apostar por activos que por su naturaleza intangible se vuelven valiosos, escasos, difíciles de imitar y difíciles de sustituir. En medio de este contexto surge lo que se conoce como capital intelectual (CI) el cual entre otras cosas puede generar una diferencia favorable entre el valor de mercado y el valor en libros de una empresa (Hall, 1993).

Sus orígenes no están definidos con exactitud, por ejemplo en el libro de Edvinson y Malone (1999) se sugiere su existencia desde principios de la humanidad, Brooking (1997) menciona que su origen va ligado al momento que un vendedor logró una buena relación con su cliente, para Fernandes (2010) es una ramificación natural de la teoría de los Recursos y Capacidades (Barney, 1991; Wernerfelt, 1984). Ya en fechas concretas, en el año 1969 por primera vez el economista John Kenneth Galbraith aborda el término CI (Bontis, 1998) para posteriormente, en 1991 Stewart con su artículo Brainpower publicado en la revista Fortune detonar su estudio (Serenko y Bontis, 2004). Esto coincide con algunos otros autores que mencionan que fueron los años noventas cuando se inició su estudio continuo (Edvinson y Malone, 1999; Roos, Bainbridge y Jacobsen, 2001).

Diversos esfuerzos pioneros se realizaron tratando de medir el CI convirtiéndose hoy en clásicos, entre estos se encuentra la empresa sueca Skandia, primer organización que realizó un esfuerzo para medir sus activos intangibles (Bontis, 2001) con su modelo Navigator (Edvinsson, 1997). Otros modelos en esta línea son el Balanced Scorecard (Kaplan y Norton, 1997), Technology Broker
(Brooking, 1997), Canadian Imperial Bank (Saint-Onge, 1996), Intangible Assets Monitor (Sveiby, 1997), Intelect (Bueno et al., 2011; Velasco y Espinoza, 2017), Intellectus (Bueno et al., 2011), entre otros, hasta llegar al presente donde modelos con técnicas más sofisticadas como ecuaciones estructurales (EE) miden la relación del CI con otras variables.

En lo que respecta a su conceptualización, para Stewart citado por Edvinsson (1997, p.369) es "algo que no puedes tocar pero puede hacerte rico", Edvinson y Malone (1999, p.32) por su parte, lo entienden como "la posesión de conocimientos, experiencia aplicada, tecnología organizacional, relaciones con clientes y destrezas profesionales que dan una ventaja competitiva en el mercado", Brooking (1997, p.9) lo define como "la combinación de activos inmateriales que permite funcionar a la empresa”, según Roos y Roos (1997, p. 415) "es la suma de los activos ocultos de la empresa que no se capturaron completamente en el balance general" y para Bontis, Janošević y Dženopoljac (2015, p.2) son "factores no físicos que contribuyen a la creación de valor en la empresa".

Con las definiciones anteriores, más las definiciones adoptadas por los autores sujetos a esta revisión de la literatura (véase apéndice A) se puede entender al CI como la suma de activos intangibles basados en el conocimiento que posee y le generan valor a la empresa. Aunque existen diferentes formas de clasificarlo (Chen, James y Chang, 2006) los capitales humano, estructural y relacional son sus componentes más aceptados en la literatura (Funes, 2007). Esta clasificación de componentes es utilizada de forma recurrente en estudios de CI-Innovación (por ejemplo: Allameh, 2018; Cabrilo y Dahms, 2018; Cheng, Xiang, Sher y Liu, 2018; Galeitzke, 
Steinhöfel, Orth y Kohl, 2017; Kianto, Sáenz y Aramburu, 2017).

De acuerdo con Bontis (1999) el capital humano es el conocimiento tácito radicado en los empleados de la empresa, el capital estructural se refiere a las rutinas organizacionales y el capital relacional es el conocimiento inmerso en las relaciones externas de la empresa. El capital humano es el componente central del CI (Iqbal, Latif, Marimon, Sahibzada y Hussain, 2019) y la tarea de convertirlo en capital estructural se convierte en el principal reto para las organizaciones ya que este último es el único capital que realmente es propiedad de la empresa y permanece en esta cuando los empleados se han retirado a sus casas (Edvinson y Malone, 1999; Roos y Roos, 1997).

La importancia del CI radica en que este representa para las empresas un impulsor de la ventaja competitiva sustentable (Mohammad, Sofian y Salmiah, 2013; Tayles, Pike y Sofian, 2007), un factor clave de crecimiento (Ansari, Barati y Sharabiani, 2016), creación de valor (Elsetouhi, Elbeltagi y Haddoud, 2015; Kianto, Sáenz y Aramburu, 2017; Roos, Bainbridge y Jacobsen, 2001; Subramaniam y Youndt, 2005), competitividad, buenos resultados (Funes, 2007), generación de utilidades, marcas, procesos, productos, creación de valor (Cabrilo y Dahms, 2018), un éxito sostenible, una mejora en desempeño corporativo (Dost, Badir, Ali y Tariq, 2016), un desempeño superior (Yuquian y Dayuan, 2015), mejora del desempeño, obtención de ventaja competitiva (Buenechea-Elberdin, 2017) y la más importante fuente de ventaja competitiva sustentable (Roos y Roos, 1997).

Una de estas ventajas competitivas que emanan del CI es la innovación (BuenecheaElberdin, 2017), esta juega un rol muy importante en las organizaciones y puede verse re- flejada en distintos beneficios entre los que se pueden mencionar crecimiento en ventas, mejora en la cuota de mercado, satisfacción de los clientes, crecimiento de la cartera de clientes, entre otros, los cuales son factores básicos de crecimiento (Ansari, Barati y Sharabiani, 2016), es decir, la innovación es un elemento critico para las organizaciones y está relacionada directamente con su resultado (Iqbal et al., 2019).

La conceptualización de la innovación en la literatura es muy diversa y no existe consenso ni definición única (BuenecheaElberdin, 2017), una referencia frecuente es Schumpeter (1934) quien la define como: a) la introducción de un bien nuevo, b) la introducción de un nuevo método de producción, c) la apertura de un nuevo mercado, d) la conquista de una nueva fuente de suministro y e) la creación de una nueva organización en cualquier industria. Según la Organización para la Cooperación y el Desarrollo Económicos (OCDE) mediante su manual de Oslo es "la implementación de un producto nuevo o significativamente mejorado (bien o servicio), o proceso, un nuevo método de mercadeo o un nuevo método organizativo en las prácticas de negocios, organización en el lugar de trabajo o relaciones externas" (OCDE, 2005, p.46).

Chesbrough (2003) hace la diferencia entre invención e innovación, aclarando que una innovación consiste en una invención implementada y llevada al mercado. De acuerdo a Drucker (1985) es la herramienta de los empresarios para generar un negocio o un servicio diferente. En esta línea, considerando las conceptualizaciones de autores contemporáneos que han abordado la relación CIInnovación (por ejemplo: BuenecheaElberdin, Kianto y Sáenz, 2018; Cabrilo y Dahms, 2018; Inchausti, 2017; Jardon, 2018) este trabajo la entiende como algo nuevo lle- 
vado a la práctica dentro de la organización que le genera un beneficio.

El CI y la innovación están estrechamente relacionados en la renovación de las empresas (Cabrilo y Dahms, 2018), específicamente el CI representa la capacidad de innovar que posee la empresa (Bontis, 1999) ya que para crear conocimiento nuevo como lo son nuevas ideas, nuevos conceptos, entre otras, se necesita un conocimiento previo (Buenechea-Elberdin, Kianto y Sáenz, 2018). En concreto, el CI es considerado generador (Nejjari y Aamoum, 2020), impulsor (Buenechea-Elberdin, 2017) y fuente de la innovación (Inchausti, 2017).

$\mathrm{Si}$ bien en la literatura existen revisiones previas de esta relación (BuenecheaElberdin, 2017; Cano, Sanchez, González y Pérez, 2014; Mention, 2012; Mohammad, Sofian y Salmiah, 2013; Nejjari y Aamoum, 2020; Zambon y Monciardini, 2015), algunas tienen cierta antigüedad de haberse realizado, otras tienen otros propósitos y algunas otras metodologías que los han llevado a una profundidad de análisis diferente al presente estudio.

Así pues, partiendo de la importancia del CI-Innovación, que no existe un estudio similar y de las implicaciones que puede generar, este trabajo se fija como objetivo una revisión de la literatura sobre la relación de ambos conceptos que contemple los años 2016, 2017, 2018 y 2019. Las tres interrogantes que soportan esta investigación son:

PI1. ¿Qué tendencias presenta el estudio de la relación del CI-Innovación del año 2016 al año 2019?

PI2. ¿Cuáles son los puntos relevantes que surgen al analizar el estudio de la relación del CI-Innovación del año 2016 al año 2019?

PI3. ¿Cuál es el escenario futuro de la relación CI-Innovación?
La respuesta a la pregunta de investigación 1 (PI1) permite saber mediante estadística descriptiva, por ejemplo: la cantidad de estudios realizados por año, el tipo de investigación que se ha llevado a cabo, quienes son los autores clásicos y contemporáneos más citados, entre otras.

Por su parte, la respuesta a la pregunta de investigación 2 (PI2) muestra los puntos que llaman la atención desde diferentes perspectivas como son aportación al conocimiento, metodología, implicaciones, entre otras.

Por último, la respuesta a la pregunta de investigación 3 (PI3) con base en las tendencias de su estudio, en revisiones de la literatura previas y en los cambios vertiginosos de la actualidad, se presenta un escenario futuro de la relación.

\section{Método}

Para cumplir el propósito de la investigación se llevó a cabo una revisión sistemática de la literatura la cual a decir de Mulrow (1994) proporciona el método más eficiente y de alta calidad para analizar literatura extensa. Tradicionalmente utilizada en ciencias médicas, recientemente a ganado popularidad en la literatura de gestión (Denyer y Neely, 2004). Se distingue de otro tipo de revisiones al adoptar un proceso replicable, riguroso, científico y transparente (Cook, Mulrow y Haynes, 1997) logrando con ello minimizar el sesgo y la subjetividad (Mention, 2012). De acuerdo a Tranfield, Denyer y Smart (2014) las fases a seguir son: definir objetivos claros y precisos; definir criterios de inclusión específicos, búsqueda de artículos relevantes en revistas predefinidas; evaluación de la calidad del estudio; síntesis de los estudios en un marco analítico explícito y por último, la presentación comprensible de la información.

En concreto, la presente investigación consistió en una revisión de la literatura 
estructurada en cuatro secciones, la primera de ellas consiste en una introducción teórica al tema, en la segunda sección se define la metodología que se llevó a cabo, seguida del análisis y discusión de los resultados y por último, en la cuarta sección se presentan las conclusiones del estudio.

\section{Generación de la muestra a analizar}

Para obtener la muestra a analizar se consideraron revisiones similares previas que abordaron el estudio de la relación CIInnovación (Buenechea-Elberdin, 2017; Cano et al., 2014; Mention, 2012; Mohammad, Sofian y Salmiah, 2013; Nejjari y Aamoum, 2020; Zambon y Monciardini, 2015) y el propósito de la presente investigación, de esta forma se seleccionaron siguiendo a Mention (2012) únicamente artículos publicados y revisados por pares con las siguientes características:

- Publicados en la Web of Science (WOS) y Scopus únicamente ya que son las dos principales bases de datos existentes (Salvador-Oliván y AgustinLaCruz, 2015). Estas dos bases de datos fueron utilizadas en revisiones previas argumentando que eran bases de datos de las más calificadas. (BuenecheaElberdin, 2017; Mention, 2012; Nejjari y Aamoum, 2020)

- $\quad$ Escritos en inglés ya que este idioma es el oficial de la academia (BuenecheaElberdin, 2017).

- $\quad$ En su título todos llevan incluidas las palabras "Intellectual Capital e Innovation", "Intellectual Capital y New product" o "Intellectual Capital y New process" buscando capturar que todos lleven en esencia la relación de CIInnovación. Estudios previos similares han coincidido en estas palabras para su selección de artículos a revisar (Buenechea-Elberdin, 2017; Mention, 2012; Nejjari y Aamoum, 2020).

- Publicados en los años 2016, 2017, 2018 y 2019. Se optó por este rango de años ya que Buenechea-Elberdin (2017) utilizó una metodología similar abarcando los años 2006 al 2015, así pues la intención es generar conocimiento nuevo.

- $\quad$ Se tomaron artículos de corte cualitativo, cuantitativo, mixto y teórico abarcando con ello todo lo publicado. Se siguió este criterio pensando en un panorama lo más completo posible.

Esta recopilación dio como resultado 56 artículos en WOS y 56 en Scopus, con los mismos se elaboró una lista en Excel eliminando los duplicados. El paso siguiente fue analizar artículo por artículo para comprobar que abordaran la relación CI-Innovación, 3 artículos fueron detectados que no entraban en esta categoría por lo que fueron eliminados restando 70 artículos para el análisis definitivo. Buenechea-Elberdin (2017) en su revisión de la literatura logró ubicar únicamente 40 artículos lo que sugiere que el estudio de la relación CI-Innovación ha incrementado.

\section{Procedimiento para tratamiento de los datos}

Con los nombres de los artículos en Excel se procedió a agregar la siguiente serie de columnas con el propósito de poder contestar las preguntas que guían esta investigación.

- Fuente: se identifica en que base de datos de las consultadas se encuentra cada artículo, las opciones son WOS, Scopus, y WOS y Scopus.

- Revista: se identifica el nombre de la revista que publicó cada trabajo. 
JCR (Journal Citation Report): aplica con un sí o un no para aquellos artículos que estén dentro de la WOS.

- $\quad$ Citas: se anota el número de citas que tenga cada trabajo.

- Tipo de investigación: en este rubro se define el tipo de la investigación.

- Técnica: en esta columna se determina la técnica empleada para cada tipo de investigación.

- Lugar de estudio: se ubica al país donde fue realizado cada trabajo.

- Industria: se refiere al tipo de industria donde se realizó el estudio.

- Muestra: se busca conocer las características más relevantes de la muestra.

- Tipo de innovación: se busca conocer cuál es la abordada por cada trabajo.

- Variable adicional: es interesante saber si la relación CI-Innovación se está relacionando con otras variables y si es así determinar cuales son.

- Clasificación del CI utilizada: se refiere a la clasificación de componentes. Se utiliza las siglas $\mathrm{CH}$ para capital humano, CE para capital estructural, CO para capital organizacional, CR para capital relacional y CS para capital social.

- Subramaniam y Youndt (2005): al ser considerados clave estos autores, se busca conocer si los artículos del año 2016 al 2019 continúan citándolos.

- Teoría: se identifica el enfoque adoptado por cada trabajo.

Adicional se agregaron otras columnas como el objetivo y las conclusiones principales, la intención es conocer lo más que sea posible de cada artículo para poder captar su esencia. Es conveniente mencionar que al estar los artículos publicados en distintas revistas estos no presentan de forma homogénea sus características; sin embargo, se pretende estandarizar los datos.

\section{Análisis y discusión de los resultados}

De acuerdo con el diseño metodológico seguido se generó una tabla presentada como apéndice A, misma que por cuestión de espacio no fue posible presentar completa ${ }^{1}$. Ahora bien, con el propósito de llevar un orden en el análisis y discusión de los resultados es necesario recordar cada pregunta de investigación:

PI1. ¿Qué tendencias presenta el estudio de la relación del CI-Innovación del año 2016 al año 2019?

La respuesta a esta pregunta se encuentra en la estadística descriptiva a que fueron sometidos los artículos (véase la tabla del apéndice A). A continuación, se analizan algunos rubros:

Autores: Si se considera únicamente al primer autor se puede percibir que 2 autores (Buenechea-Elberdin, Marta y Zhang, Min) cuentan con 3 publicaciones, de ahí le siguen 2 autores con 2 publicaciones y resto solo ha publicado en una ocasión. Lo que sugiere que la mayoría no está al 100\% enfocada en el estudio de esta relación. Es probable que muchos de ellos estudien la relación del CI con otras variables e igualmente de la innovación en su relación con otras variables.

Revista: 9 de los trabajos analizados fueron publicados en el Journal of Intellectual Capital convirtiéndola en la revista más recurrida, lo cual Agostini y Nosella (2017) confirman al señalar que la relación CIInnovación en esta revista se ha vuelto un tema de moda. Esta revista es JCR cuartil 1 lo

\footnotetext{
${ }^{1}$ En caso de necesitar consultar la tabla completa puede ponerse en contacto con el autor de este artículo.
} 
que sugiere que el tema ha sido abordado y sometido a altos estándares de calidad en lo que a revistas se refiere.

JCR: De los 70 trabajos sujetos a revisión, 54 se encuentran en revistas de la WOS y específicamente 36 son JCR, lo que habla de lo bien valorado que se encuentra el tema en la academia.

Tipo de investigación: 55 de los 70 artículos sujetos a revisión son de tipo cuantitativo, lo cual sugiere que es un tema consolidado en el que continuamente se están contrastando teorías. Esto va de la mano con lo reportado por Buenechea-Elberdin (2017).

Lugar de estudio: China considerando a Hong Kong se encuentra a la cabeza como lugar de estudio con 10 trabajos, seguida por España (6 trabajos) y Pakistán (5 trabajos). Llama la atención la actividad que tienen las economías emergentes en el estudio de esta relación no así los países más desarrollados como Estados Unidos de América (2 trabajos realizados), el Reino Unido de Gran Bretaña e Irlanda del Norte (1 trabajo realizado) o Alemania (1 trabajo realizado) por citar algunos.

Industria: la industria manufacturera es indiscutiblemente la preferida de los autores con 24 trabajos donde la consideran como muestra. Aunque está ligada a la tecnología también se pueden hacer estudios no ligadas a esta (por ejemplo, innovación de marketing o innovación organizativa). En este sentido, este trabajo coincide con lo encontrado por Buenechea-Elberdin, (2017).

Técnica: en congruencia con el tipo de investigación cuantitativa que predomina en los estudios, la técnica más utilizada son las EE con 38 trabajos misma que goza de gran aceptación en el área de la dirección estratégica (Fernandes, 2010) y en segundo lugar, se observan las regresiones. Lo anterior se presenta inverso a lo reportado por Buenechea-
Elberdin (2017) quien en su revisión de literatura reportó que las regresiones era la técnica más utilizada seguida de las EE.

Se puede mencionar también que el estudio de la relación CI-Innovación se muestra focalizada a nivel empresa con 64 trabajos considerando las revisiones de la literatura, es decir, la innovación se toma como algo nuevo en la empresa y buscando en esencia la ventaja competitiva. Las percepciones van de la mano con el uso de EE como técnica cuantitativa de tratamiento de datos (37 trabajos). La vertiente más utlizada de EE es mínimos cuadrados parciales (PLS por sus siglas en inglés) con 23 trabajos y el software favorito para su cálculo es Smartpls (10 trabajos).

La técnica del informante clave utilizada en un $30 \%$ de los trabajos es la forma de captar las percepciones de la empresa, esta consiste en que de cada empresa encuestada se selecciona una persona ${ }^{2}$ que esté involucrada en las iniciativas de innovación y a esta se le aplica un cuestionario que refleje la situación de la empresa.

Aunque existen diferentes formas de clasificar el CI (Chen, James y Chang, 2006), la clasificación de los componentes del CI más utilizada con 26 trabajos del total analizados sigue la tendencia de la literatura, es decir, capital humano, capital estructural y capital relacional (Funes, 2007).

Ahora bien, la segunda pregunta de investigación fue: PI2. ¿Cuáles son los puntos relevantes que surgen al analizar el estudio de la relación del CI-Innovación del año 2016 al año 2019?

Entre lo más relevante sobresale que todos los estudios empíricos prueban una relación positiva del CI con la innovación de alguna forma aunque existen discrepancias o puntos por aclarar en la relación, por ejemplo: Zhang, Qi, Wang, Pawar y Zhao (2018) con

\footnotetext{
${ }^{2}$ Puede ser el director general (Chief Executive Officer en inglés o CEO por sus siglas), gerente o algún mando intermedio.
} 
una misma metodología miden el efecto del CI en la innovacion de productos tanto en China como en India encontrando que el efecto directo del CI en el desempeño de la innovación del producto es significativamente mayor en China que en India. A nivel componentes, los resultados de la relación capital humano e innovación son radicalmente opuestos ya que mientras algunos autores no encuentran efecto directo del primero sobre el segundo (por ejemplo: Cabrilo y Dahms, 2018; Xu, Shang, Yu y Liu, 2019), otros estudios si encuentran un impacto positivo (por ejemplo, Agostini y Nosella, 2017; Prester, Podrug y Darabos, 2016).

Respecto a los lugares geográficos donde se ha venido estudiando el fenómeno llama la atención lo siguiente:

- África únicamente presenta un artículo realizado en Túnez. Si se considera que el CI se comporta diferente en cada país o región (Buenechea-Elberdin, 2017) y que la innovación se manifiesta en diferentes formas (Buenechea-Elberdin, Kianto y Sáenz, 2018) se hace necesario conocer el comportamiento de la relación en este escenario.

- Latinoamérica refleja actividad por medio de Brasil, Colombia, Ecuador y $\mathrm{Pa}-$ raguay, en total cinco trabajos en los cuatro años analizados que comparado con los dos trabajos encontrados por Buenechea-Elberdin (2017) habla de la importancia que se le empieza a dar en esta región al tema.

- Específicamente, en el caso de México, al ser la segunda economía de Latinoamérica y no figurar en esta lista deja en el aire la duda si se ha resignado a ser un país seguidor de innovaciones o aprovechar la vecindad con los Estados Unidos de América.

Si se parte de las siguientes tres ideas: a) que un país está compuesto por sus empresas económicamente hablando, b) que para las empresas la innovación es necesaria para ser competitivas (Costa, Fernández-Jardon Fernández y Figueroa Dorrego 2014) y c) que específicamente los países en vías de desarrollo no tienen la misma capacidad de los países desarrollados para invertir en investigación y desarrollo (ONUDI, 2016), obliga a cuestionarse ¿qué tanto están valorando y fomentando los países en vías de desarrollo la innovación?, ¿cuál es la estrategia de los países en desarrollo para obtener innovación? y por último, ¿qué futuro económico les espera en caso que no estén buscando la innovación para sus empresas?.

Por otro lado, sobresale que desde la revisión de Buenechea-Elberdin (2017) se puede observar la importancia de Subramaniam y Youndt (2005) a través de las citas recibidas $(77 \%$ de los trabajos analizados 10 citaban), estos autores sufrieron un declive en la presente revisión reduciéndose a un $68 \%$ en los trabajos analizados, esto quiere decir que su estudio sigue siendo un icono en este campo del conocimiento pero que puede estar perdiendo presencia gradualmente. En este sentido, por el número de citas destaca Kianto, Sáenz y Aramburu (2017) con su trabajo titulado Knowledge-based human resource management practices, intellectual capital and innovation, al tener 62 citas excede el doble del más inmediato siguiente, lo que sugiere que está convirtiéndose en un referente a seguir.

Aunque los artículos giran en que el CI es fuente, incentiva o propicia la innovación en cualquiera de sus modalidades, se observa 
un artículo disruptivo ${ }^{3}$, (Subramanian y Van de Vrande, 2019), este logra probar que los proyectos de desarrollo de productos nuevos ricos en CI tardan más en concluirse, lo cual da un giro en el grueso de la literatura. Algunos autores ya han dejado ver un lado negativo del CI, por ejemplo: Sveiby (1997) sugería que los activos intangibles pueden ser perjudiciales para la organización sino se gestionan o se utilizan, Harvey y Lusch (1999) hablan ya de pasivos intangibles generados que serían lo inverso al CI, este tiene efectos negativos relacionado con procesos de planificación estratégica débiles, condiciones de trabajo peligrosas, limpieza ambiental potencial, posible manipulación del producto, mala reputación corporativa, entre otras. Esta línea, aunque débil ha sido mencionada en diversas tesis doctorales (Nava, 2010; Villacorta, 2004).

En el análisis se pueden observar nuevas propuestas para los componentes del CI, por ejemplo se habla de capital de información, capital social interno y externo, capital cultural, capital social vertical, capital material, capital relacional interno y externo, competencia humana, capital empleado, capital de renovación, capital estructural interno, capital espiritual. Otro dato relevante es que un $28 \%$ de los estudios cuantitativos analizados se presentan sin contrastar una teoría, es decir, son evidencias empíricas sin contraste teórico que se enfocan en medir y establecer una relación (véase el apéndice $\mathrm{A}$ ).

La relación se muestra consolidada y de los 70 trabajos analizados, 51 introducen una variable adicional, sea como independiente, moderadora o dependiente, entre estas se puede mencionar al desempeño en diferentes modalidades ( 7 trabajos), intercambio de conocimientos (5 trabajos) y capacidad absortiva (3 trabajos). Las variables de control más utili- zadas son el tamaño, la antigüedad y la industria de las empresas.

Respecto a la innovación, los estudios la ubican generalmente como un resultado del CI en un entorno empresarial; sin embargo existen trabajos disruptivos, por ejemplo: $\mathrm{Mu}-$ rray, Papa, Cuozzo y Russo (2016) la plantean afectando al CI, es decir como variable independiente, Khan, Qing y Khan (2019) en su estudio la manejan como mediadora, Ali, Rahman, Nurdasila y Sofyan (2019) manejan la innovación a nivel gobierno, otros autores la han abordado a nivel universidades (Iqbal et al., 2019; Kousar, Zafar, Batool y Sajjad, 2019), también se ha manejado a nivel de un departamento de policía (Alshamsi, Isaac y Bhaumik, 2019a, 2019b).

Por último, la tercera pregunta fue: PI3. ¿Cuál es el escenario futuro de la relación CIInnovación?

El estudio de la relación CI-Innovación se extenderá en la medida que se amplíen las definiciones y clasificaciones tanto de CI como de innovación, así mismo, si se parte que el CI es una forma económica y segura de promover la innovación es probable que cada vez más países en vías de desarrollo la busquen por este medio. El CI y la innovación son dos variables que por su importancia han permanecido a lo largo del tiempo, existen revistas de alto impacto que se dedican exclusivamente a su estudio por lo que la relación entre ambas se observa a largo plazo.

Habiendo concluido la respuesta de las tres preguntas de investigación, este trabajo coincide de forma generalizada con revisiones similares previas; sin embargo difiere parcialmente de la conclusión de Nejjari y Aamoum (2020) quien es categórico al afirmar que el $\mathrm{CI}$ es generador de innovación en las organizaciones, mientras que esta investigación encuentra que no todos los componentes ni to-

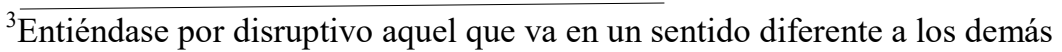


das las ocasiones procede de esta forma (Buenechea-Elberdin, Kianto y Saenz, 2018; Cabrilo y Dahms, 2018; Kianto, Sáenz y Aramburu, 2017). En este sentido, el mismo Subramaniam y Youndt (2005) encontró que el capital humano se asocia negativamente con la capacidad innovadora radical y necesita interactuar con el capital social para ejercer una relación positiva con la capacidad innovadora radical. Esta forma irregular en la influencia del CI en la innovación lleva a la conclusión de diversos autores (BuenecheaElberdin, 2017; Dost et al., 2016) en el sentido que aún falta por entender de este vínculo. Por lo anterior, parece prematuro tomar una postura definitiva, sino más bien invita a profundizar en el tema.

\section{Conclusiones}

Las revisiones de la literatura frecuentemente presentan ideas holísticas de un tema, en el caso de la presente, al usar estadística descriptiva permite identificar de una forma clara aquellos rubros específicos donde se ha focalizado el estudio y por consiguiente aquellos otros donde no se ha abordado, esto obliga a su vez a una profunda reflexión sobre sus razones y genera como consecuencia preguntas que deberán ser abordadas sea por la literatura, por las empresas o por las entidades gubernamentales correspondientes.

Es un hecho que el CI está ligado estrechamente a la innovación ya que todos los estudios cuantitativos muestran un vínculo estadístico, así mismo, si se considera que Buenechea-Elberdin (2017) en 10 años obtuvó para estudio únicamente 40 artículos para su revisión y la presente investigación en un periodo de 4 años y con una metodología similar obtuvó 70 artículos, sin lugar a dudas permite afirmar que el estudio de esta relación va en ascenso. Cada empresa que aprovecha y gestiona correctamente su CI podrá generar innovación lo cual es un antecedente de éxito. Las empresas exitosas representan para las accionistas mayores utilidades, para la sociedad más empleos y a nivel país estabilidad económica y social. Por lo anterior debe ser prioridad profundizar e impulsar desde distintos niveles, perspectivas y escenarios el estudio de la relación CI-Innovación.

Este trabajo tiene diversas implicaciones, la primera es relacionada con los investigadores que se vayan integrando al estudio del tema, ya que sirve como punto de partida y los guía hacia las lagunas existentes en la literatura. Una segunda implicación es para las empresas, ya que muestra la importancia y la forma como se relaciona el CI con la innovación. La principal limitación del presente estudio es que solo se utilizaron 2 bases de datos. Como futuras líneas de investigación se sugiere profundizar en las teorías que se están utilizando así como en las evidencias empíricas que no presenten un contraste teórico.

\section{Referencias}

Agostini, L., \& Nosella, A. (2017). Enhancing radical innovation performance through intellectual capital components. Journal of Intellectual Capital, 18(4), 789-806. doi:10.1108/JIC-10-2016-0103

Ali, H., Rahman, A., Nurdasila, D., \& Sofyan, I. (2019). Contribution of intellectual capital strategic readiness and government innovation. $O p$ ción, 35 (23), 1253-1276. Recuperado de https:// produccioncientificaluz.org/index.php/opcion/ article/view/30113/31139

Allameh, S. M. (2018). Antecedents and consequences of intellectual capital: The role of social capital, knowledge sharing and innovation. Journal of Intellectual Capital, 19(5), 858-874. doi:10.1108/JIC-05-2017-0068

Alshamsi, S., Isaac, O., \& Bhaumik, A. (2019a). Effect of transformational leadership on intellectual capi- 
tal and organizational innovation. International Journal on Emerging Technologies, 10(1), 66-76. Recuperado de https://www.researchgate.net/ publica-

tion/335465882_Effect_of_Transformational_Le adership_on_Intellectual_Capital_and_Organizat ional_Innovation

Alshamsi, S., Isaac, O., \& Bhaumik, A. (2019b). The effects of intellectual capital on organizational innovation within Abu Dhabi police in UAE. International Journal on Emerging Technologies, 10(1), 50-58. Recuperado de https:// www.researchgate.net/

publica-

tion/335465800_The_Effects_of_Intellectual_Ca pital_on_Organizational_Innovation_within_Ab u_Dhabi_Police_in_UAE

Ansari, R., Barati, A., \& Sharabiani, A. A. A. (2016). The role of dynamic capability in intellectual capital and innovative performance. International Journal of Innovation and Learning, 20(1), 4767. doi:10.1504/IJIL.2016.076671

Barney, J. (1991). Firm resources and sustained competitive advantage. Journal of Mangement, 17(1), 99-120. Recuperado de https:// josephmahoney.web.illinois.edu/BA545_Fall\% 202019/Barney\%20(1991).pdf

Bontis, N. (1998). Intellectual capital: An exploratory study that develops measure and models. Management Decision, 2(36), 63-76. doi:: 10.1108/00251749810204142

Bontis, N. (1999). Managing organizational knowledge by diagnosing intellectual capital: Framing and advancing the state of the field. International Journal of Technology Management, 18(5), 433462. doi:10.1504/ijtm.1999.002780

Bontis, N. (2001). Assessing knowledge assets: A review of the models used to measure intellectual capital. International Journal of Management Reviews, 3(1), 41-60. doi:10.1111/14682370.00053

Bontis, N., Janošević, S., \& Dženopoljac, V. (2015). Intellectual capital in serbia's hotel industry. International Journal of Contemporary Hospitality Management, 27(6), 1365-1384. doi:10.1108/ IJCHM-12-2013-0541

Brooking, A. (1997). El capital intelectual. El principal activo de las empresas del tercer milenio (Primera). Barcelona, España: Paidós.

Buenechea-Elberdin, M. (2017). Structured literature review about intellectual capital and innovation. Journal of Intellectual Capital, 18(2), 262-285. doi:10.1108/JIC-07-2016-0069

Buenechea-Elberdin, M., Kianto, A., \& Sáenz, J.
(2018). Intellectual capital drivers of product and managerial innovation in high-tech and low-tech firms. $R$ and D Management, 48(3), 290-307. doi:10.1111/radm.12271

Buenechea-Elberdin, M., Sáenz, J., \& Kianto, A. (2017). Exploring the role of human capital, renewal capital and entrepreneurial capital in innovation performance in high-tech and low-tech firms. Knowledge Management Research and Practice, 15(3), 369-379. doi:10.1057/s41275017-0069-3

Bueno, E., Del Real, H., Fernández, P., Longo, M., Merino, C., Murcia, C., \& Salmador, M. P. (2011). Modelo Intellectus: Medición y Gestión del Capital Intelectual. Madrid, España: CIC.

Bueno, E., Salmador, M. P., \& Merino, C. (2008). Génesis, concepto y desarrollo del capital intelectual en la economía del conocimiento: una reflexión sobre el modelo Intellectus y sus aplicaciones. Estudios de Economía A plicada, 26(2), 4363. Recuperado de /http://www.revista-eea.net

Cabrilo, S., \& Dahms, S. (2018). How strategic knowledge management drives intellectual capital to superior innovation and market performance. Journal of Knowledge Management, 22 (3), 621-648. doi:10.1108/JKM-07-2017-0309

Cano, M. del C., Sanchez, G. C., González, M., \& Pérez, J. (2014). El rol del capital intelectual en la innovación de las empresas. European Scientific Journal, 10(28), 348-366. Recuperado de https:// www.semanticscholar.org/paper/EL-ROL-DELCAPITAL-INTELECTUAL-EN-LAINNOVACIÓN-DE-Alvarado-Mart $\% \mathrm{C} 3 \%$ AD-

nez/40bf4602fe6cecab75cf2338119b79b09605d $\mathrm{d} 8 \mathrm{~b}$

Chen, Y. S., James, M. J., \& Chang, C. H. (2006). The influence of intellectual capital on new product development performance - The manufacturing companies of Taiwan as an example. Total Quality Management and Business Excellence, 17 (10), 1323-1339. doi:10.1080/14783360601058979

Cheng, J. S., Xiang, Y., Sher, P. J., \& Liu, C. W. (2018). Artistic intervention, intellectual capital, and service innovation: a case study of a Taiwan's hotel. Service Business, 12(1), 169-201. doi:/10.1007/s1 1628-017-0342-9

Chesbrough, H. (2003). Open Innovation. Boston, Massachusetts: Harvard Business School Publishing Corporation.

Cook, D. J., Mulrow, C. D., \& Haynes, R. B. (1997). Systematic Reviews: Synthesis of Best Evidence for Clinical Decisions. Annals of Internal Mede- 
cine, 126(5), 376-380. Recuperado de https:// www.researchgate.net/

publica-

tion/14157764_Systematic_Reviews_Synthesis of_Best_Evidence_for_Clinical_Decisions

Costa, R. V., Fernández-Jardon, C., \& Figueroa, P. (2014). Critical elements for product innovation at Portuguese innovative SMEs: An intellectual capital perspective. Knowledge Management Research and Practice, 12(3), 322-338. doi:10.1057/kmrp.2014.15

Denyer, D., \& Neely, A. (2004). Introduction to special issue: Innovation and productivity performance in the UK. International Journal of Management Reviews, 5-6(3-4), 131-135. Recuperado de doi:10.1111/j.1460-8545.2004.00100.X

Dost, M., Badir, Y. F., Ali, Z., \& Tariq, A. (2016). The impact of intellectual capital on innovation generation and adoption. Journal of Intellectual Capital, 17(4), 675-695. doi:10.1108/JIC-04-20160047

Drucker, P. (1985). The Practice of Innovation. In H. \& Row (Ed.), Innovation and Entrepreneurship Practice and Principles (pp. 19-33). Estados Unidos de América: Harper\&Row.

Edvinson, L., \& Malone, M. S. (1999). El capital intelectual: Como identificar y calcular el valor inexplotado de los recursos intangibles de su empresa. España: Gestión 2000.

Edvinsson, L. (1997). Developing intellectual capital at Skandia. Long Range Planning, 30(3), 366-373. doi:10.1016/s0024-6301(97)90248-x

Elsetouhi, A., Elbeltagi, I., \& Haddoud, M. Y. (2015). Intellectual Capital and Innovations: Is Organisational Capital a Missing Link in the Service Sector? International Journal of Innovation Management, 19(02), 1-29. doi:10.1142/ S1363919615500206

Fernandes, R. (2010). La influencia del capital intelectual en la Innovación de productos : una aplicación a pequeñas y medianas empresas innovadoras de Portugal. Tesis doctoral, Departamento de Organizacion de Empresas y Marketing Universidad de Vigo. España.

Funes, Y. (2007). Valuación de los activos intangibles el caso de UNAM. Tesis Doctoral. Universidad Nacional Autónoma de México.

Galeitzke, M., Steinhöfel, E., Orth, R., \& Kohl, H. (2017). Intellectual Capital-Driven Technology and Innovation Management. International Journal of Innovation and Technology Management, 14(5), $\quad 1750028$ doi:10.1142/ S0219877017500286
Hall, R. (1993). A framework linking intangible resources and capabilities tot sustainable competitive advantage. Strategic Management Journal, 14(8), 607-618. Recuperado de doi:10.1002/ smj.4250140804

Harvey, M. G., \& Lusch, R. F. (1999). Balancing the intellectual capital books: Intangible liabilities. European Management Journal, 17(1), 85-92. doi:10.1016/S0263-2373(98)00065-6

Inchausti, A. (2017). Determinantes del capital relacional en la innovación: una aplicación al sector de automoción español. Tesis doctoral. Universidad del Pais Vasco. España.

Iqbal, A., Latif, F., Marimon, F., Sahibzada, U. F., \& Hussain, S. (2019). From knowledge management to organizational performance: Modelling the mediating role of innovation and intellectual capital in higher education. Journal of Enterprise Information Management, 32(1), 36-59. doi:10.1108/JEIM-04-2018-0083

Jardon, C. M. (2018). Moderating effect of intellectual capital on innovativeness in Latin American subsistence small businesses. Knowledge Management Research and Practice, 16(1), 134-143. doi:10.1080/14778238.2018.1428069

Kaplan, R., \& Norton, D. (1997). Cuadro de Mando Integral.España. Gestión 2000.

Khan, S. Z., Qing, Y., \& Khan, N. U. (2019). Impact of intellectual capital management on sustainable competitive advantage via business model innovation. ACM International Conference Proceeding Series, 212-216. doi:10.1145/3312662.3312688

Kianto, A., Sáenz, J., \& Aramburu, N. (2017). Knowledge-based human resource management practices, intellectual capital and innovation. Journal of Business Research, 81(2017), 11-20. doi:10.1016/j.jbusres.2017.07.018

Kousar, S., Zafar, M., Batool, S. A., \& Sajjad, A. (2019). The mediating role of absorptive capacity in the relationship between intellectual capital and organizational innovation in higher education institutes of Punjab, Pakistan. Pakistan Journal of Commerce and Social Science, 13(3), 656-679. Recuperado de https:// www.researchgate.net/ publication/336362009_The_Mediating_Role_of_Absor ptive_Capacity_in_the_Relationship_between_In tellectual_Capital_and_Organizational_Innovatio $\mathrm{n}$ _in_Higher_Education_Institutes_of_Punjab_P akistan

Mention, A.-L. (2012). Intellectual Capital, Innovation and Performance: a Systematic Review of the 
Literature. Business and Economic Research, 2 (1), 1-37. Recuperado de doi:10.5296/ ber.v2i1.1937

Mohammad, R. K., Sofian, S., \& Salmiah, M. A. (2013). The relationship between intellectual capital and innovation: a review. International Journal of Business and Management Studies, 2 (1), 561-581. doi:10.1177/0258042X15572420

Mulrow, C. D. (1994). Rationale for systematic reviews. SystematicReviews, 309(3), 597-599. Recuperado de doi:10.2323/jgam.35.151

Murray, A., Papa, A., Cuozzo, B., \& Russo, G. (2016). Evaluating the innovation of the Internet of Things: empirical evidence from the Intellectual Capital assessment. Business Process Management Journal, 22(2), 341-356. doi:10.1108/ BPMJ-05-2015-0077

Nava, R. (2010). Modelo de capital intelectual para la UAEMex (2009) en el marco de la competitividad institucional. Tesis Doctoral. Universidad Autónoma del Estado de México.

Nejjari, Z., \& Aamoum, H. (2020). Intellectual capital as a generator of innovation in companies: A systematic review. Humanities and Social Sciences Reviews, 8(1), 464-479. doi:10.18510/ hssr.2020.8158

Organización para la Cooperación y el Desarrollo Económicos [OCDE]. (2005). Oslo Manual. Francia: OECD y doi:10.1787/9789264013100-en

Organización de las naciones unidas para el desarrollo industrial [ONUDI]. (2016). Informe sobre el Desarrollo Industrial 2016. Organización de las naciones unidas para el desarrollo industrial. Austria: Organización de las naciones unidas. Recuperado de https://www.unido.org/sites/ default/files/2015-12/

EBOOK_IDR2016_OVERVIEW_SPANISH_0. pdf

Prester, J., Podrug, N., \& Darabos, M. (2016). FourComponent Model of Intellectual Capital and its Impact on Process and Product Innovations. In Proceedings of the 8th European conference on Intellectual Capital ECIC 2016. Italia: Academic Conferences and Publishing International Limited Reading.

Roos, G., \& Roos, J. (1997). Measuring your company's intellectual performance. Long Range Planning, 30(3), 413-426. doi:10.1016/s0024-6301 (97) $90260-0$

Roos, G., Bainbridge, A., \& Jacobsen, K. (2001). Intellectual capital analysis as a strategic tool. Strate- gy \& Leadership, 29(4), 21-26. https:// doi.org/10.1108/10878570110400116

Saint-Onge, H. (1996). Tacit knowledge the key to the strategic alignment of intellectual capital. Strategy \& Leadership, 24(2), 10-16. Recuperado de https://doi.org/10.1108/eb054547

Salvador-Oliván, J., \& Agustin-LaCruz, C. (2015). Correlación entre indicadores bibliométricos en revistas de Web of Science y Scopus. Revista General de Información y Documentación, 25 (2), 341-359. Recuperado de doi:/10.5209/ rev_RGID.2015.v25.n2.51241

Schumpeter, J. A. (1934). The Theory of Economic Development: An inquiry into profits, capital, credit, interest and the business cycle. Estados Unidos de América. Harvard University Press.

Serenko, A., \& Bontis, N. (2004). Meta-review of knowledge management and intellectual capital literature: Citation impact and research productivity rankings. Knowledge and Process Management, 11(3), 185-198. doi:/10.1002/kpm.203

Subramaniam, M., \& Youndt, M. A. (2005). The Influence of Intellectual Capital on the Types of Innovative Capabilities. Academy of Management Journal, 48(3), 450-463. doi: 10.2307/20159670

Subramanian, A. M., \& van de Vrande, V. (2019). The role of intellectual capital in new product development: Can it become a liability? Journal of Operations Management, 65(6), 517-535. doi:/10.1002/joom.1045

Sveiby, K. (1997). The new organizational wealthn managing and measuring intangible asset.Estados Unidos de América. Berrett-Koehler Publishers,.

Sveiby, K. E. (1997). The Intangible Assets Monitor. Journal of Human Resource Costing \& Accounting, 2(1), 73-97. doi:/10.1108/eb029036

Tayles, M., Pike, R. H., \& Sofian, S. (2007). Intellectual capital, management accounting practices and corporate performance: Perceptions of managers. Accounting, Auditing and Accountability Journal, 20(4), 522-548. doi:10.1108/09513570710762575

Tranfield, D., Denyer, D., \& Smart, P. (2014). Towards a methodology for developing evidence informed management knowledge by means of a systematic review. British Journal of Management, 14(2), 207-222. doi:10.1080/16258312.2014.11517339

Velasco, A., \& Espinoza, J. A. (2017). A Method for the Management of Intellectual Capital in Research Centers. Revista Internacional de Gestión Del Conocimiento y La Tecnología, 5(1), 41-60. 
Recuperado de https://

www.upo.es $>$ gecontec $>$ article $>$ downland $>$ pdf

Villacorta, M. (2004). Posibles Soluciones a Problemas De La Normalización Contable. Tesis Doctoral. Universidad Complutense de Madrid.

Wernerfelt, B. (1984). A Resource-Based View of the Firm. Strategic Management Journal, 5 (2), 171180. doi: $10.2307 / 2486175$

Xu, J., Shang, Y., Yu, W., \& Liu, F. (2019). Intellectual capital, technological innovation and firm performance: Evidence from China's manufacturing sector. Sustainability (Switzerland), 11(19), 116. doi:10.3390/su11195328

Yuquian, H., \& Dayuan, L. (2015). Effects of intellectual capital on innovative performance. Management Decision, 32(3), 250-269. doi:10.1108/ S1569-3732(2011)0000014001

Zambon, S., \& Monciardini, D. (2015). Intellectual capital and innovation. A guideline for future research. Journal of Innovation Economics \& Management, 2(17), 13-26. doi:10.4324/9781315393100-12

Zhang, M., Qi, Y., Wang, Z., Pawar, K. S., \& Zhao, X. (2018). How does intellectual capital affect product innovation performance? Evidence from China and India. International Journal of Operations \& Production Management, 38(3), 895-914. doi:10.1108/IJOPM-10-2016-0612 


\section{Apéndice A}

Tabla 1.

Relación de artículos analizados

\begin{tabular}{|c|c|c|c|c|c|c|}
\hline Título & Año & $\begin{array}{c}\text { Tipo de } \\
\text { investiga- } \\
\text { ción }\end{array}$ & $\begin{array}{l}\text { Lugar de } \\
\text { estudio }\end{array}$ & Industria & Tipo de Innovación & $\begin{array}{l}\text { Variable adicio- } \\
\text { nal }\end{array}$ \\
\hline $\begin{array}{l}\text { An Intellectual Capital perspective for Business Model } \\
\text { Innovation in technology-intensive industries: empiri- } \\
\text { cal evidences from Italian spin-offs. }\end{array}$ & 2017 & Cualitativa & Italia & $\begin{array}{l}\text { Spin-offs } \\
\text { tecnológicas }\end{array}$ & $\begin{array}{l}\text { Innovación de mode- } \\
\text { lo de negocio. }\end{array}$ & No aplica. \\
\hline $\begin{array}{l}\text { Antecedents and consequences of intellectual capital: } \\
\text { The role of social capital, knowledge sharing and inno- } \\
\text { vation. }\end{array}$ & 2018 & Cuantitativa & Irán & Hotelería & $\begin{array}{l}\text { En una variable inclu- } \\
\text { yó diversas dimensio- } \\
\text { nes. }\end{array}$ & $\begin{array}{l}\text { Intercambio de } \\
\text { conocimientos y } \\
\text { Capital social }\end{array}$ \\
\hline $\begin{array}{l}\text { Artistic intervention, intellectual capital, and service } \\
\text { innovation: a case study of a Taiwan's hotel. }\end{array}$ & 2017 & Cualitativa & Taiwán & Hotelería & $\begin{array}{l}\text { Innovación de servi- } \\
\text { cio. }\end{array}$ & $\begin{array}{l}\text { Intervención artís- } \\
\text { tica. }\end{array}$ \\
\hline $\begin{array}{l}\text { Building small firm performance through intellectual } \\
\text { capital development: Exploring innovation as the } \\
\text { "black box". }\end{array}$ & 2018 & Cuantitativa & $\begin{array}{l}\text { Estados } \\
\text { Unidos de } \\
\text { América }\end{array}$ & $\begin{array}{c}\text { Diversa } \\
\text { (manufacturera } \\
\text { y servicios) }\end{array}$ & $\begin{array}{l}\text { En una variable inclu- } \\
\text { yó innovación radical } \\
\text { e incremental para } \\
\text { productos o servicios. }\end{array}$ & $\begin{array}{l}\text { Desempeño orga- } \\
\text { nizacional. }\end{array}$ \\
\hline $\begin{array}{l}\text { Components of intellectual capital a focus toward the } \\
\text { innovation of organizations. }\end{array}$ & 2017 & $\begin{array}{c}\text { Teórico } \\
\text { (Documental } \\
\quad \text { ) }\end{array}$ & No aplica & No aplica & No aplica. & No aplica. \\
\hline $\begin{array}{l}\text { Contribution of intellectual capital strategic readiness } \\
\text { and government innovation. }\end{array}$ & 2019 & Cuantitativa & Indonesia & Gubernamental & $\begin{array}{l}\text { Capacidad de Innova- } \\
\text { ción del gobierno. }\end{array}$ & $\begin{array}{l}\text { Sistemas de traba- } \\
\text { jo de alto desem- } \\
\text { peño, Desempeño } \\
\text { del Gobierno. }\end{array}$ \\
\hline $\begin{array}{l}\text { Creating competitive advantage: Linking perspectives } \\
\text { of organization learning, innovation behavior and } \\
\text { intellectual capital. }\end{array}$ & 2017 & Cuantitativa & China & hotelería & Conducta innovadora. & $\begin{array}{l}\text { Ventaja competiti- } \\
\text { va, Aprendizaje } \\
\text { organizacional. }\end{array}$ \\
\hline $\begin{array}{l}\text { Does intellectual capital allow improving innovation } \\
\text { performance? A quantitative analysis in the SME con- } \\
\text { text. }\end{array}$ & 2017 & Cuantitativa & Italia & Manufacturera & $\begin{array}{l}\text { En una variable inclu- } \\
\text { yó diversas dimensio- } \\
\text { nes. }\end{array}$ & No aplica. \\
\hline $\begin{array}{l}\text { Effect of transformational leadership on intellectual } \\
\text { capital and organizational innovation. }\end{array}$ & 2019 & Cuantitativa & $\begin{array}{l}\text { Emiratos } \\
\text { Árabes } \\
\text { Unidos }\end{array}$ & $\begin{array}{l}\text { Departamento } \\
\text { de policía de } \\
\text { Abu Dhabi }\end{array}$ & $\begin{array}{l}\text { Innovación organiza- } \\
\text { cional. }\end{array}$ & $\begin{array}{l}\text { Liderazgo trans- } \\
\text { formacional. }\end{array}$ \\
\hline $\begin{array}{l}\text { Effects of intellectual capital and university knowledge } \\
\text { in indgenous innovation: evidence from indian SMEs. }\end{array}$ & 2019 & Cuantitativa & India & Manufacturera & Innovación indígena. & $\begin{array}{l}\text { Conocimiento } \\
\text { universitario. } \\
\text { Integración multi- }\end{array}$ \\
\hline Enhancing intellectual capital for e-service innovation. & 2016 & Cuantitativa & Taiwán & $\begin{array}{c}\text { Hotelería y } \\
\text { financiera }\end{array}$ & $\begin{array}{l}\text { Innovación de servi- } \\
\text { cio por internet. }\end{array}$ & $\begin{array}{l}\text { funcional. } \\
\text { Competencia } \\
\text { colaborativa exter- } \\
\text { na. }\end{array}$ \\
\hline $\begin{array}{l}\text { Enhancing radical innovation performance through } \\
\text { intellectual capital components. }\end{array}$ & 2017 & Cuantitativa & Itaia & Manufacturera & $\begin{array}{l}\text { Desempeño de la } \\
\text { innovación radical. }\end{array}$ & No aplica. \\
\hline $\begin{array}{l}\text { Evaluating the innovation of the Internet of Things } \\
\text { Empirical evidence from the intellectual capital assess- } \\
\text { ment. }\end{array}$ & 2016 & Mixta & $\begin{array}{l}\text { No aplica } \\
\text { (utiliza } \\
\text { bases de } \\
\text { datos) }\end{array}$ & $\begin{array}{l}\text { Tecnologías de } \\
\text { la información }\end{array}$ & $\begin{array}{l}\text { Innovación de la } \\
\text { internet. }\end{array}$ & No aplica. \\
\hline $\begin{array}{l}\text { Exploring corporate disclosure and reporting of intel- } \\
\text { lectual capital (IC): emerging innovations Introduction. }\end{array}$ & 2017 & $\begin{array}{l}\text { Teórico } \\
\text { (Análisis de } \\
\text { artículos) }\end{array}$ & No aplica & No aplica & $\begin{array}{l}\text { Innovaciones emer- } \\
\text { gentes. }\end{array}$ & No aplica. \\
\hline $\begin{array}{l}\text { Exploring dynamic capabilities, intellectual capital and } \\
\text { innovation performance relationship: Evidence from } \\
\text { the garment manufacturing. }\end{array}$ & 2019 & Cuantitativa & Indonesia & $\begin{array}{l}\text { Manufacturera } \\
\text { de prendas }\end{array}$ & $\begin{array}{l}\text { En una variable inclu- } \\
\text { yó diversas dimensio- } \\
\text { nes. }\end{array}$ & $\begin{array}{l}\text { Capacidades Diná- } \\
\text { micas. }\end{array}$ \\
\hline $\begin{array}{l}\text { Four component model of intellectual capital and its } \\
\text { impact on process and product innovations. }\end{array}$ & 2016 & Cuantitativa & 16 países & Manufacturera & $\begin{array}{l}\text { Innovación de pro- } \\
\text { ducto y de procesos. }\end{array}$ & No aplica. \\
\hline $\begin{array}{l}\text { From Knowledge management to organizational per- } \\
\text { formance Modelling the mediating role of innovation } \\
\text { and intellectual capital in higher education. }\end{array}$ & 2018 & Cuantitativa & Pakistán & $\begin{array}{c}\text { Educación } \\
\text { (universidades) }\end{array}$ & $\begin{array}{l}\text { Innovación de la } \\
\text { educación. }\end{array}$ & $\begin{array}{l}\text { Gestión del cono- } \\
\text { cimiento y Desem- } \\
\text { peño organizacio- }\end{array}$ \\
\hline
\end{tabular}




\section{Tabla 1.}

\section{Relación de artículos analizados (continuación)}

How does intellectual capital affect product innovation performance? Evidence from China and India.

How intellectual capital, knowledge management, and the business environment affect thailand's food industry innovation.

How strategic knowledge management drives intellectual capital to superior innovation and market performance.

Impact of intellectual Capital Management on Sustainable Competitive Advantage via Business Model Innovation.

Impact of intellectual capital on innovation capability and organizational performance: an empirical investigation.

Impacts of intellectual capital on process innovation and mass customisation capability: direct and mediating effects.

Imperatives of intellectual capital and technical skills for innovation: perspective on higher education.

Innovation, intellectual capital and competitiveness in small companies in southern Colombia.

Intellectual capital and radical innovation: Exploring the quadratic effects in technology-based manufacturing firms.

Intellectual capital and technological innovation of companies.

Intellectual capital drivers of product and managerial innovation in high-tech and low-tech firms.

Intellectual capital efficiency, technological innovation and family management.

Intellectual capital for exploratory and exploitative innovation Exploring linear and quadratic effects in construction contractor firms.

Intellectual capital-based innovation planning: empirical studies using wiNK model.

2016

Cuantitativa

Intellectual Capital-Driven Technology and Innovation Management.

\section{7}

Cuantitativa Alemania

Intellectual Capital-Enhancing HR, Absorptive Capacity, and Innovation.

2018

Cuantitativa

China e

Cuantitativa Serbia

2016

Cuantitativa

España

2016

Cuantitativa

China

España y Portugal
Manufacturera

Innovación de producto. alimentos con propiedad intelectual

Manufacturera y de servicios

En una variable incluyó diversas dimensiones (producto, proceso y tecnología).

En una variable incluyó diversas dimensiones.

PYME (No especifica)

Innovación de modelo de negocio.

Capacidad de innovación.

nnovación sos.

$$
\text { No aplica. }
$$

Diferentes sectores econó-

micos

(servicios, financieros, producción)

Manufacturera

Diversa

Empresas

manufactureras $\mathrm{y}$ de servicios

Manufactureras

Empresas contratistas de construcción con 50 o más empleados.

Tecnologías de

la Información

y la comunicación (TIC)

Manufacturera y servicios

Adaptabilidad de la cadena de suministro e Integración del conocimiento del proveedor.

Gestión del conocimiento y Entorno de los negocios.

Desempeño de mercado, Gestión estratégica del conocimiento.

Ventaja competitiva sustentable.

En una variable incluyó diversas dimensiones.

Innovación radical.

Innovación tecnológica.

Innovación de producto/ servicio e innovación gerencial. Innovación de productos y procesos como una sola variable.

Innovación estratégica exploratoria y de explotación.

\section{Desempeño de la} innovación.

Innovación de producto y de procesos.

Desempeño de la

Diferentes

Cuantitativa Australia industrias y información que incluye Innovación exploratoria e Innovación explotadora.
No aplica.

Capacidad de personalización masiva.

Habilidades técnicas.

Competitividad.

Subsidio del gobierno.

No aplica.

Gestión familiar.

No aplica.

No aplica.

No aplica.

Prácticas de recursos humanos

y Capacidad

absortiva. 
Tabla 1.

Relación de artículos analizados (continuación)

Intellectual capital, absorptive capacity and product innovation.

Intellectual capital, entreprenurial orientation, and technical innovation in small and medium-sized enterprises.

Intellectual Capital, Innovation and Firm Performance of Pharmaceuticals: A Study of the London Stock Exchange.

Intellectual Capital, Innovation, and Performance: Empirical Evidence from SMEs.

Intellectual Capital, Knowledge Sharing and Innovation

Performance: Evidence form the chinese Construction

Industry.

Intellectual capital, organisational climate, innovation culture, and SME performance Evidence form Croatia.

Intellectual Capital, Technological Innovation and Firm Performance: Evidence from China's Manufacturing Sector.

Intellectual capital's influence on small innovation enterprises' innovation capacity based on the enterprises' life cycle.

Investigating the mediating role of knowledge sharing in the relationship between intellectual capital and organizational innovation (A case study of Iranian software companies).

Joint forces: Towards an integration of intellectual capital theory and the open innovation paradigm.

Knowledge management strategies, intellectual capital, and innovation performance: a comparison between high- and low-tech firms.

Knowledge-based human resource management practices, intellectual capital and innovation.

leveraging Suppliers for product innovation performance: The moderating role of Intellectual Capital.

Linking knowledge sharing, intellectual capital and social capital to innovation performance.

Linking national business system and firm level innovation: A serial mediation analysis with intellectual capital and absorptive capacity.

Linking technology innovation strategy, intellectual capital and technology innovation performance in manufacturing SMEs.

Management of intellectual capital and innovation.

Perceptions shrimp exporters.

Organizational innovation based on intellectual capital and transfer tacit knowledge.

Personal Values and Entrepreneurial attitude as Intellectual Capital : Impact Innovation in Small Enterprises.

2017

Cuantitativa

Brasil

Diversas Innovación de pro-
ducto.

2019 Cuantitativa Jordania

PYME (No especifica)

2017 Cuantitativa Inglaterra Farmacéutica

2015 Cuantitativa Austria

Manufacturera

2019 Cuantitativa

China

Industria de la construcción

2018 Cuantitativa

Croacia

Manufacturera $\mathrm{y}$ de servicios

2019 Cuantitativa

China

Manufacturera

Cuantitativa

China

Diversa

Cuantitativa

Irán

Software

2019

Cuantitativa

España

Manufacturera

Cuantitativa

España

No especifica

2017 Cuantitativa

España

Manufacturera y de servicios

2018

Cuantitativa

10 países

Teórico

No aplica

No aplica

201

201

Cuantitativa

Italia

201

Cualitativa Ecuador Camaronera

201

Cuantitativa Indonesia Textil (Batik)

2018
Cuantitativa Paraguay
Innovación de pro-
En una variable incluyó diversas dimensiones.

Innovación técnica.

En una variable incluyó diversas dimensiones. ducto.

En una variable incluyó diversas dimensiones.

Cultura de la Innovación.

Innovación tecnológica.

Capacidad de innovación.

Innovación organizacional.

Innovación abierta.

Lo maneja como una sola variable llamada innovación de empresas.

Innovación de producto.

\section{Desempeño de la} innovación.

Nivel de la Innovación de la empresa (radical e incremental).

Desempeño de la Innovación tecnológica incremental y radical.

Evidencias de Innovación

En una variable incluyó diversas dimensiones.

En una variable incluyó diversas dimensiones.

\section{Intercambio de} conocimientos.

Capacidad absortiva.

Orientación emprendedora la empresa.

Desempeño de la empresa

Intercambio de

Clima organi-

zacional y

Desempeño de las Pyme.

Desempeño de la empresa.

No aplica.

No aplica.

Estrategias de gestión del conocimiento. Prácticas de gestión de recursos humanos basadas en el conocimiento.

Apalancamiento de proveedores.

Intercambio de conocimientos y Capital social.

Sistema nacional de negocios

y Capacidad absortiva.

Estrategia de innovación tecnológica.

Las prácticas de gestión organizacional.

Transferencia

del conoci-

miento tácito.

No aplica.
Desempeño de conocimientos. 
Tabla 1.

Relación de artículos analizados (continuación)

Redefining the relationship between intellectual capital and innovation: The mediating role of absorptive capacity.

Research on the relationship among intellectual capital, learning from failures and enterprise innovation performance.

Structured literature review about intellectual capital and innovation.

Studying the mediating role of knowledge sharing and market orientation to enhance the intellectual capital effect on the organizational innovation (supervision of insurance companies in Lorestan Province).

The effect of enterprise social networks use on exploitative and exploratory innovations Mediating effect of subdimensions of intellectual capital.

The effects of intellectual capital on organizational innovation within Abu Dhabi police in UAE.

The impact of intellectual capital and knowledge flows on incremental and radical innovation: Empirical findings form a transition economy of Vietnam.

The impact of intellectual capital on innovation generation and adoption.

The impact of intellectual capital on innovation via the mediating role of knowledge management: A structural equation modelling approach.

The impact of intellectual capital, organizational capabilities and innovation on firm performance of textile sector: a moderating effect of GSP Plus.

The importance of accountability for innovation development viewed through the mediating role of intellectual capital.

The influence innovation strategy and company's financial performance mediated by intellectual capital and internal process performance

The mediating role of absorptive capacity in the relationship between intellectual capital and organizational innovation in higher education institutes of Punjab, Pakistan.

The Path to innovation: The antecedent perspective of Intellectual Capital and OrganizationalCharacter.

The role of intellectual capital in new product development: Can it become a liability?.

The role of intellectual capital in the intrapreneurship process and its effects on the innovation radicalness.

Understanding the nexus of intellectual, social and psychological capital towards business innovation through critical insights from organizational culture.

What comes first? A model of intellectual capital management, strategic innovation and novel business model interaction.
2016

2018

Teórico

No aplica

Revisión de la literatura

No aplica

2016

Cuantitativa

Irán

Aseguradoras

Tecnologías de la información

2019 Cuantitativa Túnez

y la comunicación (TIC)

Árabes

Unidos

2018

Cuantitativa

Vietnam

Departamento

de policía de

Abu Dhabi

Diversa

(manufacturera

y servicios)

2016

Cuantitativa

No lo menciona

Industria quÍmica

2017 Cuantitativa Jordania

Telecomunicación

Textil

2019 Cuantitativa Pakistán

Teórico

No aplica

No aplica

Indonesia

Manufacturera

Institutos de educación

2019 Cuantitativa Pakistán superior (11

públicas y 14 privadas)

Diversas in-

dustrias con

2018 Cuantitativa China certificación de alta y no alta tecnología.

Base de

datos

2019 Cuantitativa (Estados

Unidos de

Biotecnología

América)

2017

Teórico

No aplica

No aplica

Teórico

No aplica No aplica

Utilizó a Tesla,

Teórico (conceptual)
No aplica Apple, Face-

book y Ama-
En una variable incluyó diversas dimensiones.

Capacidad absortiva.

\section{Desempeño de la} Innovación.

Aprendizaje fallido.

No aplica.

No aplica.

Intercambio de conocimientos y

Orientación al mercado.

El Uso de las redes sociales empresariales.

Innovación organizacional (producto, proceso y administrativa).

Innovaciones incremental y radical.

Flujos de conocimiento ascendentes y descenden-

tes.

Generación y adopción de Innovación.

En una sola contemplo radical e incremental.

En una variable incluyó diversas dimensiones.

\section{Desarrollo de la} Innovación.

Estrategia de innovación.

Innovación organizacional (nuevo método, nueva regulación y nuevas prácticas administrativas).

Innovación Tecnológica e Innovación en el modelo de negocios.

Desarrollo de nuevos productos.

Innovación radical. Emprendimiento.

Capital sicológi-

Innovación de nego- co, Capital Social cio. y Cultura organizacional.

Innovación estratégi- Modelo de Negoca. cio. 